\title{
Possible effects on avionics induced by terrestrial gamma-ray flashes
}

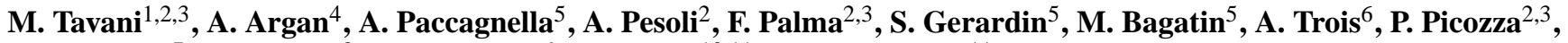 \\ P. Benvenuti ${ }^{7}$, E. Flamini ${ }^{8}$, M. Marisaldi ${ }^{9}$, C. Pittori ${ }^{10,11}$, and P. Giommi ${ }^{11}$ \\ ${ }^{1}$ INAF-IAPS, via del Fosso del Cavaliere 100, 00133 Rome, Italy \\ ${ }^{2}$ University of Rome Tor Vergata, Department of Physics, via della Ricerca Scientifica 1, 00133, Rome, Italy \\ ${ }^{3}$ INFN Roma “Tor Vergata", via della Ricerca Scientifica 1, 00133 Rome, Italy \\ ${ }^{4}$ INAF, Viale del Parco Mellini 84, Rome, Italy \\ ${ }^{5}$ University of Padova, Department of Information Engineering, Via Gradenigo 6B, 35131 Padova, Italy \\ ${ }^{6}$ INAF-Osservatorio Astronomico di Cagliari, Località Poggio dei Pini, Strada 54, 09012 Capoterra, Italy \\ ${ }^{7}$ University of Padova, Department of Physics and Astronomy “G. Galilei”, vicolo dell' Osservatorio 3, 35122, Padova, Italy \\ ${ }^{8}$ Agenzia Spaziale Italiana, viale Liegi 26, 00198 Rome, Italy \\ ${ }^{9}$ INAF-IASF, via Gobetti 101, 40129 Bologna, Italy \\ ${ }^{10}$ INAF Osservatorio Astronomico di Roma, via di Frascati 33, 00040 Monte Porzio Catone, Italy \\ ${ }^{11}$ ASI Science Data Center, Via E. Fermi 45, 00044 Frascati (Rome), Italy \\ Correspondence to: M. Tavani (tavani@iasf-roma.inaf.it)
}

Received: 12 November 2012 - Published in Nat. Hazards Earth Syst. Sci. Discuss.: Revised: - Accepted: 10 January 2013 - Published: 30 April 2013

\begin{abstract}
Terrestrial gamma-ray flashes (TGFs) are impulsive (intrinsically sub-millisecond) events associated with lightning in powerful thunderstorms. TGFs turn out to be very powerful natural accelerators known to accelerate particles and generate radiation up to hundreds of $\mathrm{MeV}$ energies. The number ratio of TGFs over normal lightning has been measured in tropical regions to be near $10^{-4}$. We address in this Article the issue of the possible susceptibility of typical aircraft electronics exposed to TGF particle, gamma ray and neutron irradiation. We consider possible scenarios regarding the intensity, the duration, and geometry of TGFs influencing nearby aircraft, and study their effects on electronic equipment. We calculate, for different assumptions, the total dose and the dose-rate, and estimate single-event-effects. We find that in addition to the electromagnetic component (electrons/positrons, gamma rays) also secondary neutrons produced by gamma-ray photo production in the aircraft structure substantially contribute to single-event effects in critical semiconductors components. Depending on the physical characteristics and geometry, TGFs may deliver a large flux of neutrons within a few milliseconds in an aircraft. This flux is calculated to be orders of magnitude larger than the natural
\end{abstract}

cosmic-ray background, and may constitute a serious hazard to aircraft electronic equipment. We present a series of numerical simulations supporting our conclusions. Our results suggest the necessity of dedicated measurement campaigns addressing the radiative and particle environment of aircraft near or within thunderstorms.

\section{Introduction}

Commercial aircraft are designed in compliance with specifications for natural radiation levels. In particular, aircraft have to sustain both electromagnetic discharges caused by lightning (e.g. Fisher et al., 1999; Rakov and Uman, 2005), and the natural radiation levels at cruise altitudes (IEC, 2006). However, a new class of extreme atmospheric events leads us to reconsider a possible impact on airborne vehicles. We address in this article the issue of the possible susceptibility of aircraft equipment to photon and particle irradiation caused by terrestrial gamma-ray flashes (TGFs). TGFs (currently detected in the range $100 \mathrm{keV}-100 \mathrm{MeV}$ by high-energy astrophysics satellites; Fishman et al., 1994; Smith et al., 2005; 
Marisaldi et al., 2010a,b; Briggs et al., 2010; Tavani et al., 2011; Fishman et al., 2011) are impulsive atmospheric events characterised by a great acceleration power. They usually occur in powerful thunderstorms, and have been associated mostly with intra-cloud discharges at altitudes in the range of 10-20 km (e.g. Lu et al., 2010). The TGF geographical distribution, for the most favorable detection conditions occurring in tropical regions (Smith et al., 2010), closely follows the lightning distribution (e.g. Christian et al., 2003). The average TGF occurrence has been locally determined in several geographical regions, resulting in an average number ratio $\mathcal{R} \simeq 10^{-4}$ of TGFs vs. lightning in the equatorial region (Fuschino et al., 2011).

TGFs impulsively accelerate particles in large numbers (electrons and positrons whenever they are produced by pair creation processes). The total number of accelerated electrons $N$ can reach $10^{17}-10^{18}$ or even larger values, (e.g. Dwyer et al., 2005). These particles efficiently radiate by bremsstrahlung producing a remarkably hard spectrum that currently is known up to energies near $100 \mathrm{MeV}$ (Tavani et al., 2011). It is important to note that $100 \mathrm{MV}$ is the typical maximal voltage drop between cloud-ground and inter-cloud locations separated by km-sized distances (Marshall et al., 2011; Stolzenburg et al., 2007). TGFs are then observed to accelerate particles up to the maximum voltage available in thunderclouds. It is currently not known whether acceleration occurs on a step-leader size $(50-100 \mathrm{~m})$ or larger. A variety of hypotheses have been proposed for the TGF production mechanisms (e.g. Gurevich et al., 1992; Roussel-Dupré et al., 1996; Dwyer, 2003; Carlson et al., 2007; Gurevich et al., 2006). Satellite observations, that are significantly affected by Compton scattering delays in the atmosphere, indicate a typical observed TGF duration $\tau_{\mathrm{TGF}}$ within $200 \mu$ s (Fishman et al., 2011). Taking into account the expected Compton scattering for different production atmospheric depths (typically in the range $10-20 \mathrm{~km}$ ), and the measured time delays of order of $\delta t \sim 100 \mu \mathrm{s}$ in the energy ranges $20-60 \mathrm{keV}$ and $320 \mathrm{keV}-20 \mathrm{MeV}$ (Ostgaard et al., 2008), an upper limit on the production site has been established as $h \leq 20 \mathrm{~km}$. Currently, no direct association between a typical TGF and a specific lightning substructure has been identified. We define the intrinsic TGF duration at the source as $\tau_{\mathrm{s}}=\tau_{-5} \times 10^{-5} \mathrm{~s}$, and scale our rate estimates accordingly.

We consider in this paper the main effects induced by particle and gamma-ray irradiation of an aircraft flying within the TGF radiative channel. Particles and photons are expected to interact with the aircraft structure, and produce two types of secondaries: (1) an electromagnetic component (gamma rays and electrons/positrons) resulting from the e.m. interactions in the aircraft structure (particle bremsstrahlung, electron/positron pair production, electron/positron and gamma-ray attenuation, etc.); (2) a neutron component, produced by the interaction of gamma rays with the aircraft structure. These two high-energy components deposit energy in the avionics structure and enclosed volume.
In principle, these secondaries give rise to four categories of radiation effects: (A) Total ionizing dose (TID) effects, due to the cumulative effect of ionizing particles, which cause charge trapping and interface state generation in dielectric layers. TID effects eventually lead to parametric failure of integrated circuits. (B) Displacement damage (DD) effects, due to the cumulative effects of ionizing and non-ionizing particles displacing semiconductor lattice atoms, causing parametric variations. (C) Dose rate (DR) effects, produced by intense bursts of ionizing radiation delivered within a few tens of microseconds, which give rise to large photocurrents. DR effects may lead to upsets, burn-out, and latch-up. (D) Single event effects (SEE), due to a single ionizing particle hitting a sensitive region of a device. These various types of SEEs are single event upsets, single event latch-up, etc.

Given the TGF timescale together with the flux and spectral characteristics of the possible irradiation, the impact on aircraft equipment is necessarily the result of the combined effects (A)-(D). This fact constitutes a challenge to current studies and testing of aircraft irradiation effects. The unique feature of the phenomenon addressed here is the quasisimultaneous irradiation of extended parts of the aircraft electronic equipment. In this paper we address irradiation issues without a global assessment of the combined simultaneous effects on the electronics, a study that goes beyond the capability of current testing and available data. The effects of TGF irradiation on human personnel in airborne vehicles has been addressed in Dwyer et al. (2010). Our work differs from Dwyer et al. (2010) in several aspects: (1) we consider the updated TGF gamma-ray spectrum measured up to $100 \mathrm{MeV}$ (Tavani et al., 2011); (2) we address not only the electromagnetic component but also the relevant neutron component; and (3) we focus on the impacts on the electronic equipment rather than on humans.

Our study is a first step towards a complete evaluation of the issue regarding the radiation and particle interaction on aircraft induced by a special class of very energetic lightning. Our simulations are aimed at identifying the critical parameter space for the physical quantities affecting the phenomenon (TGF intensity, duration, geometry and particle/radiation properties). A second step would be implemented by aircraft measurements capable of detecting impulsive particle and radiation effects near or within thunderstorms.

\section{The TGF radiation environment}

A TGF produces a primary flux of electrons (and positrons) and gamma rays inside a lightning radiation/discharge channel. An aircraft struck by a TGF-lighting is assumed to be affected by a portion of the overall complex lightning channel. We define the TGF active channel as the cylindrical region where most of the accelerated particles irradiate the aircraft along its axis in a front-to-back aircraft configuration. 
The longitudinal $(L)$ and radial extent $(R)$ of the TGF active channel can be deduced from observations of lightning (Rakov and Uman, 2005) and TGF timing properties (Nemiroff et al., 1997; Fishman et al., 2011). For our purposes, we can take $L \geq 50-100 \mathrm{~m}$, and $R \simeq 1-100 \mathrm{~m}$. In this paper we assume that the aircraft is within the TGF active region, most likely because of aircraft-induced lightning discharge (as discussed in Fisher et al., 1999).

The total number of radiating electrons accelerated by a TGF is normalised as $N_{\mathrm{e}}=N_{17} \times 10^{17}$, with average particle energy $\bar{E}_{\mathrm{e}}=\bar{E}_{\gamma} / \epsilon_{\mathrm{B}}$, where $\bar{E}_{\gamma}$ is the average gamma-ray energy (here assumed to be $\overline{E_{\gamma}}=10 \mathrm{MeV}$ from AGILE observations), and $\epsilon_{\mathrm{B}} \simeq 0.1-0.5$ is the bremsstrahlung efficiency for photon production by relativistic electrons. In the following, we use the average electron energy $\overline{E_{\mathrm{e}}}=10 \mathrm{MeV}, \bar{E}_{\mathrm{e}, 1}$. The TGF accelerated particles and gamma rays are assumed to be uniformly spread out over a circular cross section $A=$ $\pi R^{2}$. The electron energy fluence is then $F_{E}=N_{\mathrm{e}} \bar{E}_{\mathrm{e}} / A \simeq$ $10^{12} N_{17} \bar{E}_{\mathrm{e}, 1} / \pi R_{3}^{2} \mathrm{MeV} \mathrm{cm}^{-2}$, where we have assumed a cylindrical outer radius $R=10 \mathrm{~m}$, i.e. $R=10^{3} R_{3} \mathrm{~cm}$. Accelerated particles radiate by bremsstrahlung and the photon number fluence is then $F_{\gamma}=N_{\mathrm{e}} / A=10^{17} \epsilon^{\prime} / \pi 10^{6} R_{3}^{2} \simeq$ $3 \times 10^{10} N_{17} R_{3}^{-2} \mathrm{~cm}^{-2}$, where $\epsilon^{\prime}$ is the fractional probability of bremsstrahlung photon production within the volume and timescales of interest (we assume $\epsilon^{\prime} \sim 1$ ). Clearly, smaller values of $R$ correspond to larger radiation fluences ${ }^{1}$.

In this paper we consider the average TGF primary spectrum of electrons deduced by the AGILE high-energy observations (Tavani et al., 2011; Tavani, 2011). In particular, the non-trivial high-energy spectral component between $10 \mathrm{MeV}$ and $100 \mathrm{MeV}$ is included in the calculation for the first time (previous calculations have assumed a quite small component radiated above $10 \mathrm{MeV}$ for an exponentially cutoff electron distribution with a cutoff energy $E_{\mathrm{c}} \simeq 7 \mathrm{MeV}$, as expected from RREA calculations (e.g. Dwyer, 2003). The new energetic component corresponds to about $10 \%$ of the total energy of TGF accelerated particles. Electrons/positrons and gamma-rays interact with the avionic structure and are partially attenuated and reprocessed by the $\mathrm{Al}, \mathrm{C}$, and other heavier element material. A flux of secondary particles and gamma rays is then able to propagate inside the aircraft environment. We distinguish two main components: (1) an electromagnetic component (electrons/positrons and gamma rays); (2) a neutron component produced by photoproduction processes.

The electromagnetic component is made of (i) penetrating electrons/positrons, (ii) penetrating gamma rays; (iii) secondary gamma rays produced by bremsstrahlung, (iv) secondary electrons/positrons produced by gamma-ray pair production. We performed extensive numerical simulations of the e.m. component interaction with a typical aircraft structure using the Geant4 package (Agostinelli et al., 2003). In

\footnotetext{
${ }^{1}$ The probability of aircraft strike may be weakly dependent on $R$ because of aircraft-induced lightning (Rakov and Uman, 2005).
}

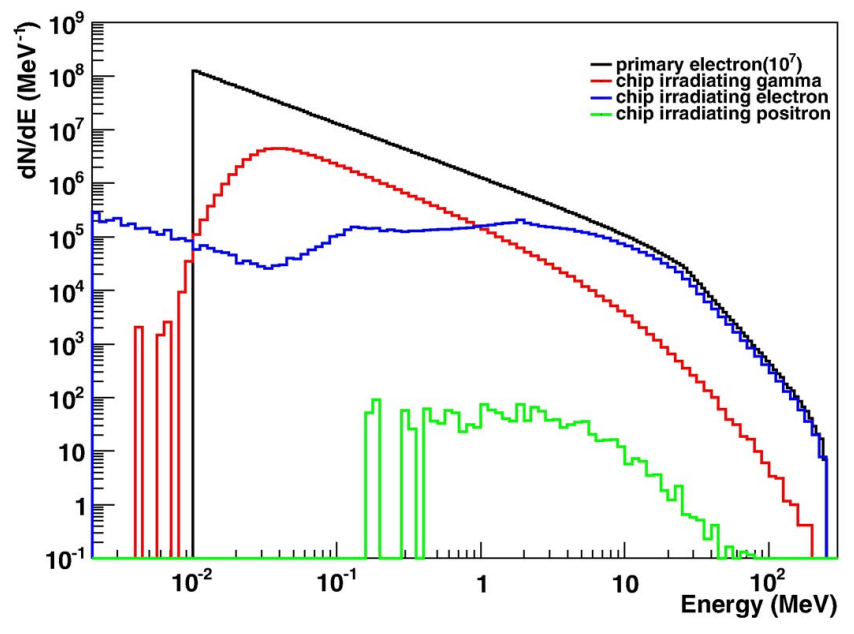

Fig. 1. An example of a possible avionics equipment irradiation for an aircraft exposed to a flux of incoming electrons. The black curve represents a possible power-law electron fluence spectrum for an effective TGF strength $X_{\mathrm{TGF}}=N_{17} R_{3}^{-2}=10^{-4}$. The coloured curves show the secondary particle fluences (electrons, positrons, and gamma rays) produced after having penetrated an equivalent aluminum shielding layer of $0.5 \mathrm{~cm}$.

particular, we studied the energy deposited by the TGF e.m. component in typical Si layers (of $500 \mu$ thickness) representing the active part of a SRAM or FPGA or similar devices. We considered different shielding configurations for a radiation attenuation equivalent Al layer of $l$ : (1) practically unshielded equipment $(l=0)$ to be applicable to devices positioned outside the aircraft frame with no significant radiation protection; (2) shielded equipment with equivalent aluminum layer thickness $l$ of different values. Figure 1 shows the result of a typical calculation of the transport of primary and secondary e.m. components reaching a Si electronic device placed inside the aircraft structure with $l=0.5 \mathrm{~cm}$. We find that the total dose accumulated in the $l_{0.5}$-shielded $\mathrm{Si}$ components of the electronic equipment, is $D_{\text {em }} \simeq(200 \mathrm{rad}) N_{17} R_{3}^{-2}$. For unshielded components, the accumulated total dose is $\sim 30 \%$ larger. This total dose, for a target within the TGF channel, is accumulated within $\tau_{\mathrm{s}}$. The dose rate expected by TGF-induced irradiation of avionic electronic components is

$\dot{D}_{\mathrm{em}} \simeq\left(2 \times 10^{7} \mathrm{rad} \mathrm{s}^{-1}\right) N_{17} R_{3}^{-2} \tau_{-5}^{-1}$.

Neutrons $(n)$ are produced by energetic gamma rays $(\gamma)$ in the energy range above $\sim 15 \mathrm{MeV}$ by the reaction $\gamma+{ }^{27} \mathrm{Al} \rightarrow{ }^{27} \mathrm{Al}^{*} \rightarrow{ }^{26} \mathrm{Al}+n$ where ${ }^{27} \mathrm{Al}^{*}$ is an excited metastable state of ${ }^{27} \mathrm{Al}$. Similar reactions occur on the other elements of the avionics structure, and in particular on carbon composite materials, $\gamma+{ }^{12} \mathrm{C} \rightarrow{ }^{12} \mathrm{C}^{*} \rightarrow{ }^{11} \mathrm{C}+n$. A typical cross-section for these photo production reactions is $\sigma \simeq 10^{-2}$ barn. These reactions are of the greatest importance and are considered here for the first time in the context 
of determining the effect of TGF-irradiation in aircraft (see, e.g. Carlson et al., 2010; Babich et al., 2008 for the TGFinduced production of neutrons in the atmosphere).

We simulated the photo production of neutrons by the TGF-induced irradiation of aircraft by a typical gamma-ray primary spectrum. We approximated the penetrated layer of material by an effective layer of aluminum of depth $L=$ $(1 \mathrm{~cm}) L_{0}$. Incoming gamma rays of energies in the range $15-40 \mathrm{MeV}$ are the most effective for interactions on aluminum. We can easily estimate the probability of prompt neutron photo production, $P=\sigma n_{\mathrm{Al}} L \simeq 6 \times 10^{-4} L_{0}$. The red curve in Fig. 2 shows the prompt photo-produced neutron (PPN) energy spectrum as resulting from our Geant 4 simulation for the incoming gamma-ray spectrum given by the black curve. The PPN fluence can be obtained from Fig. 2 by integrating over the relevant neutron kinetic energy range and by integrating in time over $1 \mathrm{~s}$. The resulting fluence is $F_{\mathrm{n}} \simeq\left(10^{7} \mathrm{~cm}^{-2}\right) N_{17} R_{3}^{-2} L_{0}$. The PPN flux (fluence per unit time), within the intrinsic TGF duration timescale $\tau_{\mathrm{s}}$ is then

$\dot{F}_{\mathrm{n}} \simeq\left(10^{10} \mathrm{~cm}^{-2} \mathrm{~s}^{-1}\right) N_{17} R_{3}^{-2} L_{0} \tau_{-3}^{-1}$,

where we assumed a millisecond scale for the PPN burst. The typical energy of the prompt neutron spectrum is $\bar{E}_{\mathrm{n}} \simeq$ $7 \mathrm{MeV}$. Neutron thermalization in the aircraft structure and interior follows.

\section{Possible radiation and particle effects}

For low/moderate values of TGF intensities implying relatively low doses and neutron fluences, displacement damage and total ionizing dose effects are not the crucial elements for electronic failures. However, they may be relevant for extreme values of the TGF radiation parameters. The electron/gamma-ray total dose can reach the critical value of $D_{\mathrm{c}}=5 \times 10^{3} \mathrm{rad}(\mathrm{Si})$ for which some very sensitive electronic components have been to shown to fail in slow irradiation regimes. In these cases, the critical combination of TGF parameters is $N_{17} R_{3}^{-2} \sim 25$. For these TGF values and conditions, critical radiation levels can be reached also for humans (Dwyer et al., 2010). In the following, we will focus on TGF-radiation induced dose rate, and single event effects. Dose rate effects on electronic equipment can be produced by the very rapid and collective effects caused by extremely strong irradiation of electronic components within a timescale shorter than a few milliseconds (US Department of Defence, 2008). Prompt pulses of Xrays and gamma rays can cause circuit upsets, latchup and burnout if delivered within timescales of tens of microseconds. Dose rate effects on electronic devices induced by the TGF delivery of a very fast and intense electron/gamma-ray dose in the aircraft equipment is possible for TGF source timescales in the range $10<\tau_{\mathrm{s}}<100 \mu \mathrm{s}$. Assuming a critical level for malfunctions (e.g. US Department of Defence,

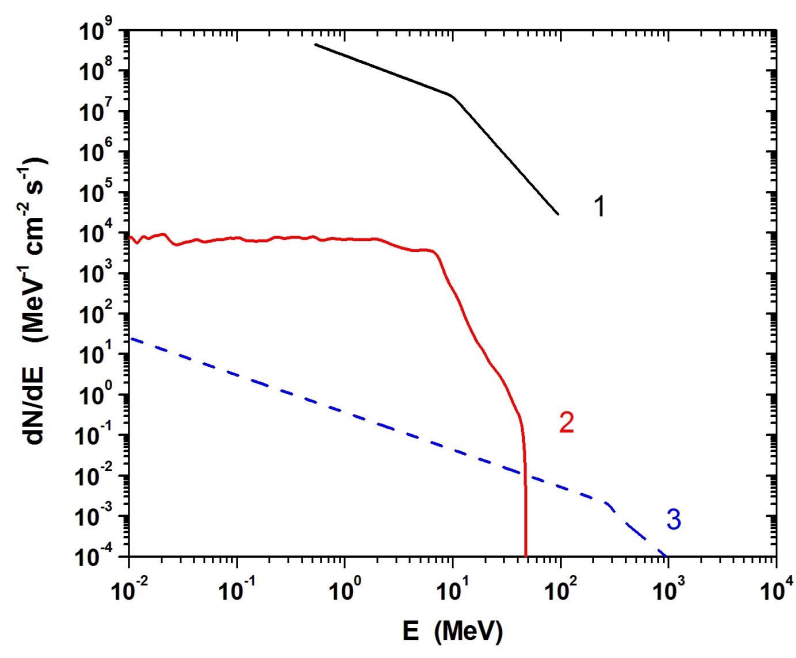

Fig. 2. An example of TGF irradiation of aircraft avionics producing photo production of secondary neutrons within the aircraft structure. Black curve (labelled “1"): primary TGF gamma-ray spectrum averaged over 1 second for a TGF strength parameter (see text) $X_{\mathrm{TGF}}=N_{17} R_{3}^{-2}=10^{-2}$. The primary spectrum is represented as a combination of two power-law components of indices $\alpha_{1}=1$ and $\alpha_{2}=3$, and a break at $E=10 \mathrm{MeV}$, as indicated by the AGILE observations. Red curve (labelled "2"): photo produced neutrons. Blue dashed curve (labelled " 3 "): energy spectrum of the natural background of atmospheric neutrons at the altitude of $12000 \mathrm{~m}$ (40000 feet) (IEC, 2006).

2008) $\dot{D}_{\mathrm{c}}=10^{8} \mathrm{rad}(\mathrm{Si}) \mathrm{s}^{-1}$, we obtain the condition for the TGF dose rate malfunctions

$N_{17} R_{3}^{-2} \tau_{-5}^{-1} \sim 5$.

Single event effects on aircraft electronics are mostly due to neutrons (e.g. Normand et al., 2006). In case of TGF irradiation, we estimate the probability of a SEU induced by prompt photo-produced neutrons on benchmark electronic equipment such as SRAMs approximating the upset process as being "slow-and-cumulative" (a complete treatment of the simultaneous effect of a large number of prompt neutrons released within a few hundredths of milliseconds inducing neutron bit upset is beyond the scope of this paper and is left to additional investigations). From IEC (2006) (see also Normand et al., 2006), we obtain the single-event-upset (SEU) cross section per bit of commercially available 4 Mbit SRAMs (see Fig. 8 of IEC, 2006) for neutron energies around $10 \mathrm{MeV}$

$$
\begin{aligned}
\sigma(\mathrm{SEU} \text { cross-section/bit }) & \simeq \alpha 3 \times 10^{-13} \mathrm{~cm}^{2} \\
\sigma(\mathrm{SEU} \text { cross-section/device }) & \simeq \alpha \times 10^{-6} \mathrm{~cm}^{2},
\end{aligned}
$$

where $\alpha(0<\alpha<1)$ takes into account the measurement scatter for different SRAM manufacturers. From Eq. (5) and the natural neutron fluence at typical aircraft altitudes (IEC, 2006), we obtain the probability of a SEU per device induced by the natural neutron background during a 10-h flight, 
Table 1. Susceptibility to TGFs.

\begin{tabular}{ll}
\hline TGF strength & Radiation effects \\
\hline$\alpha X L_{o}>0.1$ & significant neutron-induced SEEs \\
$X \geq 25$ & total dose effects in sensitive components \\
$X \tau_{-5}^{-1} \sim 5$ & dose rate effects \\
\hline
\end{tabular}

$P_{\mathrm{n}}($ natural background, $10 \mathrm{~h}) \simeq 0.07 \alpha$. On the other hand, for TGF events from the TGF-neutron fluence estimated above, we can evaluate the SEU probability $P_{\text {prompt }}$ per SRAM device induced by the TGF prompt photo-produced neutrons (PPNs)

$P_{\text {prompt }}($ TGF-PPNs $) \simeq 10 \alpha N_{17} R_{3}^{-2} L_{0}$.

A value of $P_{\text {prompt }}$ of order unity (indicative of systemic electronic failure within the auto-correction capability of current devices, as also indicated by the expected average error/Mbit) can be reached for a parameter combination $\alpha N_{17} R_{3}^{-2} L_{0} \sim 0.1$ that can occur for intense TGFs and typical structure interaction. Given the geometry and intensity of TGF irradiation, we expect susceptibility and malfunctioning for a large number of electronic devices simultaneously affected by the neutron burst. Depending on internal redundance and protection, the overall aircraft electronic system can possibly be influenced in the absence of special recovery devices/strategies. We conclude that TGF PPNs can strongly increase the probability of electronic equipment upsets during a TGF discharge, leading to the possibility of multiple failures and equipment malfunctioning.

\section{Conclusions}

In this paper we considered the conditions under which radiative and particle interaction effects can induce critical effects on current standard electronic equipment of commercial aircraft. Depending on the TGF radiative intensity, as a function of increasing "TGF fluence strength" given by the parameter combination $X=N_{17} R_{3}^{-2}$, we can distinguish two main effects due to the electromagnetic and neutron component, respectively. For e.m. processes, the relevant quantity is $X_{\mathrm{em}}=X$, whereas for neutron-induced effects the relevant quantity is $X_{\mathrm{n}}=\alpha X L_{o}$. We summarize the most relevant events in Table 1. For intense TGFs the induced radiation and particle effects on the aircraft electronic equipment are expected to increase the failure rates.

We find that a critical TGF radiative fluence strength is reached for the value

$X_{\mathrm{TGF}}=N_{17} R_{3}^{-2} \sim 1$.

Both electron/gamma ray and neutron components copiously produced inside an aircraft by TGF irradiation can induce systemic failures of the electronic system. Intense TGFs

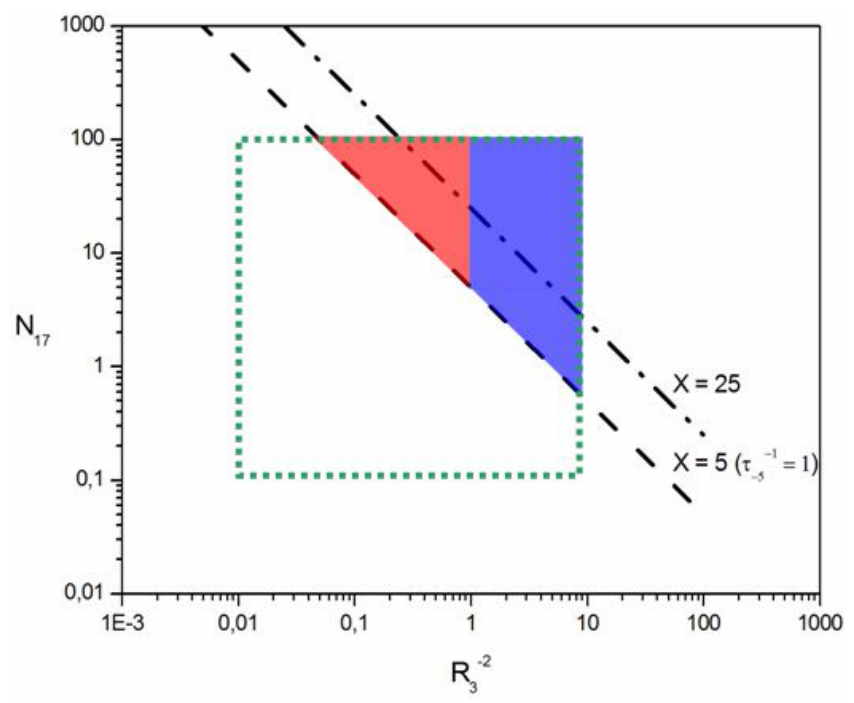

Fig. 3. Parameter space for the possible influence of TGFs on aircraft equipment for electromagnetic irradiation (electrons, positrons, gamma-rays): the total TGF particle number $N_{17}$ vs. the inverse of the squared radius of the TGF active channel $R_{3}^{-2}$, where $N_{17}$ is the particle number in units of $10^{17}$, and $R_{3}$ is the channel radius in units of $10^{3} \mathrm{~cm}$. The area marked within the dotted green lines represents the inferred combination of TGF parameters as described in the text.

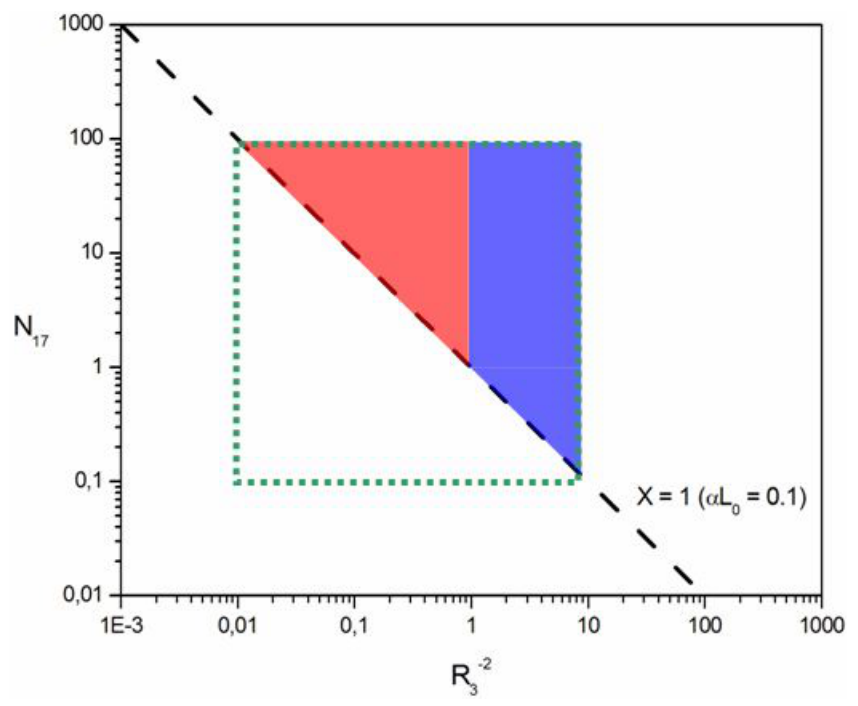

Fig. 4. Parameter space for the possible influence of TGFs on aircraft equipment for neutron (PPN) irradiation: the total TGF particle number $N_{17}$ vs. the inverse of the squared radius of the TGF active channel $R_{3}^{-2}$, where $N_{17}$ is the particle number in units of $10^{17}$, and $R_{3}$ is the channel radius in units of $10^{3} \mathrm{~cm}$. Symbol and colour definitions as in Fig. 3.

are expected to satisfy Eq. (7) under quite general conditions. Figures 3 and 4 graphically show the relevant parameter space where we assumed a TGF active channel radius 
between $\sim 3$ and $100 \mathrm{~m}$. Typical TGFs detected from space have $N_{17}$ in the range $\sim 2-10$ (e.g. Dwyer et al., 2005; Ostgaard et al., 2008). For extreme TGFs $N_{17}$ can reach a value near 100. In Figs. 3 and 4, the areas marked in red show the critical regions for TGF active channel radii of $10 \mathrm{~m}$, or larger. The blue areas show the possible critical regions for TGF active channel radii between $\sim 3$ and $10 \mathrm{~m}$ (corresponding to $R_{3}^{-2}$ between $\sim 10$ and 1 ).

TGF effects on aircraft electronic equipment at cruise altitudes (IEC, 2006) require a careful study that goes beyond the analysis based on standard particle and radiation conditions. We find that neutrons may play a crucial role (it is also interesting to note that neutrons are detected on the ground by several groups in association with thunderstorms; Shah et al., 1985; Shyam et al., 1999; Chilingarian et al., 2010; Gurevich et al., 2012). Our study identified the critical parameter space for the physical quantities affecting the possible TGF/aircraft interaction (TGF intensity, duration, geometry and particle/radiation properties). A proper assessment of the likelihood of an aircraft being affected by a TGF radiation channel should be evaluated based on meteorological and aircraft data.

We consider these calculations a first step towards a complete evaluation of the issue. At the moment, there is no systematic and satisfactory study of impulsive radiative and particle phenomena caused by lightning. Furthermore, the TGF properties (very intense photon/particle fluxes, and large neutron pulses expected close or within the discharge channel) require an analysis of aircraft electronic susceptibility that goes beyond the standard one. In order to address these issues, a second step would be implemented by aircraft measurements capable of detecting impulsive particle and radiative effects near or within thunderstorms (e.g. Smith et al., 2011). Existing data of radiation monitors operating in aircraft can also be valuable in studying impulsive events. We strongly advocate measurement campaigns along the lines suggested in this paper.

Acknowledgements. This investigation was carried out with partial support under ASI contracts nos. I/089/06/2, and I/042/10/0.

Edited by: A. Mugnai

Reviewed by: C. S. Dyer and one anonymous referee

\section{References}

Agostinelli, S., Allison, J., Amako, K. et al.: GEANT4: A Simulation toolkit, Nucl. Instr. Methods A, 506, 250-303, doi:10.1016/S0168-9002(03)01368-8, 2003.

Babich, L. P., Kudryavtsev, A. Y., Kudryavtseva, M. L., and Kutsyk, I. M.: Atmospheric gamma-ray and neutron flashes, J. Exp. Theor. Phys., 106, 65-76, doi:10.1134/S1063776108010056, 2008.

Briggs, M. S., Fishman, G. J., Connaughton, V., Bhat, P. N., Paciesas, W. S., Preece, R. D., Wilson-Hodge, C., Chaplin, V. L.,
Kippen, R. M., von Kienlin, A., Meegan, C. A., Bissaldi, E., Dwyer, J. R., Smith, D. M., Holzworth, R. H., Grove, J. E., and Chekhtman, A.: First results on terrestrial gamma ray flashes from the Fermi Gamma-ray Burst Monitor, J. Geophys. Res., 115, A07323, doi:10.1029/2009JA015242, 2010.

Carlson, B. E., Lehtinen, N. G., and Inan, U. S.: Constraints on terrestrial gamma ray flash production from satellite observation, Geophys. Res. Lett., 34, L08809, doi:10.1029/2006GL029229, 2007.

Carlson, B. E., Lehtinen, N. G., and Inan, U. S.: Neutron production in terrestrial gamma ray flashes, J. Geophys. Res., 115, A00E19, doi:10.1029/2009JA014696, 2010.

Chilingarian, A., Daryan, A., Arakelyan, K., Hovhannisyan, A., Mailyan, B., Melkumyan, L., Hovsepyan, G., Chilingaryan, S., Reymers, A., and Vanyan, L.: Ground-based observations of thunderstorm-correlated fluxes of high-energy electrons, gamma rays, and neutrons, Phys. Rev. D, 82, 043009, doi:10.1103/PhysRevD.82.043009, 2010.

Christian, H. J., Blakeslee, R. J., Boccippio, D. J., Boeck, W. L., Buechler, D. E., Driscoll, K. T., Goodman, S. J., Hall, J. M., Koshak, W. J., Mach, D. M., and Stewart, M. F.: Global frequency and distribution of lightning as observed from space by the Optical Transient Detector, J. Geophys. Res., 108, 4005, doi:10.1029/2002JD002347, 2003.

Dwyer, J. R.: A fundamental limit on electric fields in air, Geophys. Res. Lett., 30, 2055, doi:10.1029/2003GL017781, 2003.

Dwyer, J. R. and Smith, D. M.: A comparison between Monte Carlo simulations of runaway breakdown and terrestrial gammaray flash observations, Geophys. Res. Lett., 32, L22804, doi:10.1029/2005GL023848, 2005.

Dwyer, J. R., Smith, D. M., Uman, M. A., Saleh, Z., Grefenstette, B., Hazelton, B., and Rassoul, H. K.: Estimation of the fluence of high-energy electron bursts produced by thunderclouds and the resulting radiation doses received in aircraft, J. Geophys. Res., 115, D09206, doi:10.1029/2009JD012039, 2010.

Fisher, F. A., Plumer, J. A., and Perala, R. A.: Lightning protection of aircraft, Lightning Technologies Inc., Pittsfield, MA (USA), 1999.

Fishman, G. J., Bhat, P. N., Mallozzi, R., Horack, J. M., Koshut, T., Kouveliotou, C., Pendleton, G. N., Meegan, C. A., Wilson, R. B., Paciesas, W. S., Goodman, S. J., and Christian, H. J.: Discovery of Intense Gamma-Ray Flashes of Atmospheric Origin, Science, 264, 1313-1316, 1994.

Fishman, G. J., Briggs, M. S., Connaughton, V., Bhat, P. N., Paciesas, W. S., von Kienlin, A., Wilson-Hodge, C., Kippen, R. M., Preece, R., Meegan, C. A., and Greiner, J.: Temporal properties of the terrestrial gamma-ray flashes from the Gamma-Ray Burst Monitor on the Fermi Observatory, J. Geophys. Res., 116, A07304, doi:10.1029/2010JA016084, 2011.

Fuschino, F., Marisaldi, M., Labanti, C. et al.: High spatial resolution correlation of AGILE TGFs and global lightning activity above the equatorial belt, Geophys. Res. Lett., 38, L14806, doi:10.1029/2011GL047817, 2011.

Gurevich, A. V., Milikh, G. M., and Roussel-Dupré, R.: Runaway electron mechanism of air breakdown and preconditioning during a thunderstorm, Phys. Lett. A, 165, 463-468, 1992.

Gurevich, A. V., Zybin, K., and Medvedev, Y.: Amplification and nonlinear modification of runaway breakdown, Phys. Lett. A, 349, 331-339, 2006. 
Gurevich, A. V., Antonova, V. P., Chubenko, A. P., Karashtin, A. N., Mitko, G. G., Ptitsyn, M. O., Ryabov, V. A., Shepetov, A. L., Shlyugaev, Yu. V., Vildanova, L. I., and Zybin, K. P.: Strong Flux of Low-Energy Neutrons Produced by Thunderstorms, Phys. Rev. Lett., 108, 125001, doi:10.1103/PhysRevLett.108.125001, 2012.

IEC Technical Specification: Process management for avionics Atmospheric radiation effects - Accomodation of atmospheric radiation effects via single event effects within avionics electronic equipment, IEC TS 62396-1, 2006.

Lu, G., Blakeslee, R. J., Li, J., Smith, D. M., Shao, X. M., McCaul, E. W., Buechler, D. E., Christian, H. J., Hall, J. M., and Cummer, S. A.: Lightning mapping observation of a terrestrial gamma-ray flash, Geophys. Res. Lett., 37, L11806, doi:10.1029/2010GL043494, 2010.

Marisaldi, M., Fuschino, F., Labanti, C. et al.: Detection of terrestrial gamma ray flashes up to $40 \mathrm{MeV}$ by the AGILE satellite, J. Geophys. Res., 115, A00E13, doi:10.1103/PhysRevLett.105.128501, 2010a.

Marisaldi, M., Argan, A., Trois, A. et a.: Gamma-Ray Localization of Terrestrial Gamma-Ray Flashes, J. Geophys. Res., Phys. Rev. Lett., 105, 128501, doi:10.1029/2009JA014502, 2010 b.

Marshall, T. C. and Stonzelburg, M.: Voltages inside and just above thunderstorms, J. Geophys. Res., 106, 4757-4768, 2011.

Nemiroff, R., Bonnell, J., and Norris, J.: Temporal and spectral characteristics of terrestrial gamma flashes, J. Geophys. Res., 102, 9659-9665, 1997.

Normand, E., Vranish, K., Sheets, A., Stitt, M., and Kim, R.: Quantifying the Double-Sided Neutron SEU Threat, From Low Energy (Thermal) and High Energy $(>10 \mathrm{MeV})$ Neutrons, IEEE Trans. Nuclear Sci., 53, 3587-3595, 2006.

Ostgaard, N., Gjesteland, T., Stadsnes, J., Connell, P. H., and Carlson, B.: Production altitude and time delays of the terrestrial gamma flashes: Revisiting the Burst and Transient Source Experiment spectra, J. Geophys. Res., 113, A02307, doi:10.1029/2007JA012618, 2008.
Rakov, V. A. and Uman, M. A.: Lightning: physics and effects, Cambridge University Press, 2005.

Roussel-Dupré, R. and Gurevich, A. V.: On runaway breakdown and upward propagating discharges, J. Geophys. Res., 101, 2297 2311, 1996.

Shah, G. N., Razdan, H., Bhat, C. L., and Ali, Q. M.: Neutron generation in lightning bolts, Nature, 313, 773-775, 1985.

Shyam, A. and Kaushik, T. C.: Observation of neutron bursts associated with atmospheric lightning discharge, J. Geophys. Res., 104, 6867-6869, 1999.

Smith, D. M., Lopez, L. I., Lin, R. P., and Barrington-Leigh, C. P.: Terrestrial Gamma-Ray Flashes Observed up to $20 \mathrm{MeV}$, Science, 307, 1085-1088, 2005.

Smith, D. M., Hazelton, B. J., Grefenstette, B. W., Dwyer, J. R., Holzworth, R. H., and Lay, E. H.: Terrestrial gamma ray flashes correlated to storm phase and tropopause height, J. Geophys. Res., 115, A00E49, doi:10.1029/2009JA014853, 2010.

Smith, D. M., Dwyer, J. R., Hazelton, B. J., Grefenstette, B. W., Martinez-McKinney, G. F. M., Zhang, Z. Y., Lowell, A. W., Kelley, N. A., Splitt, M. E., Lazarus, S. M., Ulrich, W., Schaal, M., Saleh, Z. H., Cramer, E., Rassoul, H., Cummer, S. A., Lu, G., Shao, X.-M., Ho, C., Hamlin, T., Blakeslee, R. J., and Heckman, S.: A terrestrial gamma ray flash observed by an aircraft, J. Geophys. Res., 116, D20124, doi:10.1029/2011JD016252, 2011.

Stolzenburg, M., Marshall, T. C., Rust, W. D., Bruning, E., MacGorma, D. R., and Hamlin, T.: Electric field values observed near lightning flash initiations, Geophys. Res. Lett., 34, L04804, doi:10.1029/2006GL028777, 2007.

Tavani, M.: An update on the AGILE observations of Terrestrial Gamma-Ray Flashes, contributed talk at the 2011 AGU Fall Meeting, San Francisco (CA), 5-9 December, 2011.

Tavani, M., Marisaldi, M., Labanti, C. et al.: Terrestrial GammaRay Flashes as Powerful Particle Accelerators, Phys. Rev. Lett., 106, 018501, doi:10.1103/PhysRevLett.106.018501, 2011.

US Department of Defence: Militarily Critical Technologies List, Section 20, February 2008. 